\title{
Análisis comparativo de los pacientes con y sin trastorno mental diagnosticable.
}

A comparative study of individuals with or without mental disorder diagnosable.

\author{
Carolina Lozano Serrano a , Alberto Ortiz Lobo ${ }^{\text {b }}$, Rocío González González ${ }^{\text {c }}$
}

${ }^{a}$ Psiquiatra, Centro de Salud Mental de Leganés, Madrid. ${ }^{b}$ Psiquiatra, Centro de Salud Mental de Salamanca, Madrid. ${ }^{c}$ CAIBER, Agencia Pedro Laín Entralgo, Madrid.

Correspondencia: Carolina Lozano Serrano (carolinalozser@gmail.com)

Recibido: 30/10/2010; aceptado con modificaciones: 02/02/2011

RESUMEN: Objetivo: Analizar las diferencias entre las variables sociodemográficas, asistenciales y clínicas de estas dos poblaciones.

Metodología: Diseño: descriptivo comparativo. Ámbito: área sanitaria urbana. Población: todos los pacientes derivados al Centro de Salud Mental durante un año (n =1187). Variables: sociodemográficas, clínicas y asistenciales que se recogieron mediante una entrevista clínica y los tests GHQ-28, SCL-90-R y una escala de expectativas.

Resultados: Entre los trastornos mentales hay más solteros, menor nivel educativo, son diagnosticados con mayor frecuencia por profesionales mujeres y derivados como preferentes. Además presentan más antecedentes psiquiátricos, acuden al centro con un tratamiento psicofarmacológico pautado en mayor medida y tras la evaluación son dados de alta en menos ocasiones que los códigos Z. Los trastornos mentales puntúan más alto en todos los índices estudiados del SCL-90-R. Además señalaban que los acontecimientos vitales estaban fuera de control en más ocasiones que los códigos $\mathrm{Z}$ en el momento de la consulta.

Conclusiones: Las diferencias sociodemográficas entre ambas poblaciones son muy discretas al igual que en los estudios previos. En nuestro trabajo ambos grupos de pacientes tienen menos contactos ambulatorios previos que en estudios internacionales, pero más medicación pautada en el momento de la consulta, sobre todo en el caso de los códigos Z. Parece que los trastornos mentales tienen menos capacidades o habilidades de afrontamiento de las circunstancias adversas y son por tanto más vulnerables que los pacientes diagnosticados de código $Z$.

PALABRAS CLAVE: Códigos Z, trastornos mentales, estudio comparativo, acontecimiento vital estresante y SCL-90R.
ABSTRACT: Objective: To analyze the differences between socio-demographic variables, inpatients and outpatients of individuals assessed in a community mental health center (CMHC) with or with no diagnosable mental disorders according to ICD-10 (mental disorder or $\mathrm{Z}$ code).

Method: Design: comparative description. Scope: urban area. Population: all patients referred to the Salamanca Mental Health Service ( $n=1187$ ). Variables: sociodemographics, clinical and health service utilization gathered through clinical interview, GHQ-28, SCL-90-R and an expectations scale.

Results: Among the mental patients there are more singles, a lower level of education, they are diagnosed by female professionals more frequently, and they have priority referals. Moreover they have mental health history more frequently, they arrive at the mental health service with psychopharmacological treatment administered often and after the evaluation they are discharged less frequently than $\mathrm{Z}$ code. Mental disorders are given more weight in all the SCL-90-R indexes studied. Vital occurrences were out of control more often in mental disorders than $\mathrm{Z}$ codes at the time of evaluation.

Conclusions: The socio-demographic differences between both populations are finite like in previous studies. In our research both patient groups have fewer clinical visits than in international studies, but more prescribed medication at the time of the evaluation, especially in $\mathrm{Z}$ codes. It appears that mental patients lack social skills or the ability to stressful life events and, therefore, are more vulnerable than $\mathrm{Z}$ codes.

KEY WORDS: Z code conditions, psychiatric outpatients, comparative study, stressful life event and SCL-90R. 


\section{Introducción}

Desde hace ya algunos años se ha puesto de manifiesto el aumento en la demanda asistencial en los Servicios de Salud Mental (1, 2, 3). Gran parte de este incremento se debe a diferentes problemas que podrían catalogarse de malestares o sentimientos derivados de los avatares de la vida cotidiana y que no llegan a constituir un trastorno mental (4). Las clasificaciones nosográficas actuales, DSMIV (5) y CIE-10 (6), los denominan códigos Z y los recogen en un capítulo aparte, fuera de los trastornos mentales, denominado "Factores que influyen en el estado de salud y en el contacto con los servicios de salud".

Para explicar el fenómeno del aumento de la demanda de tratamiento del malestar en los servicios de salud mental se pueden utilizar argumentos de distinta índole. De un lado, aquellos más positivistas consideran que es el resultado de un progreso de la ciencia en cuanto a detección e intervenciones precoces de la misma manera que le ha sucedido a otras especialidades clínicas. Una manifestación de esta interpretación se puede ver reflejada en el aumento de las categorías de los trastornos mentales (de 106 en el DSM-I de 1952 a 357 en el DSM-IV). Esta perspectiva también se refleja en la ampliación de las condiciones psicológicas tratables, con conceptos como los trastornos subumbrales (que no llegan a cumplir criterios de trastorno mental) o los estados prodrómicos (personas presuntamente sanas a las que se les supone en riesgo de enfermar en el futuro) $(7,8,9,10)$

Por otro lado, están los que entienden este aumento de la demanda como parte de un proceso de medicalización común a todas las especialidades médicas, mediante el cual aspectos del sufrimiento humano son resignificados como enfermedades (11). El proceso de medicalización en salud mental o de psicopatologización es complejo y se interpreta como el resultado de una conjunción de factores corporativos (el conjunto de los técnicos en salud mental, especialmente psiquiatras y psicólogos), de intereses económicos (industria farmacéutica, empresas sanitarias) (12) y de orden social administrativo y cultural (gestión social de las poblaciones de riesgo y gestión de lo íntimo) $(13,14,15)$.

Desde la década de los 80 y 90 han aparecido varios estudios que describen un cambio en la población que consultaba en los centros de salud mental $(16,17$, $18,19,20)$ con un incremento en la asistencia a pacientes leves que limitaba la atención a los pacientes mentales más graves $(21,22,23,24,25,26)$. Además, desde la administración y los propios médicos, se ha planteado si es viable económicamente y apropiado continuar con una atención similar para este tipo de pacientes con los recursos disponibles. $(20,21,27,28)$.

A pesar del interés creciente en este tema, son pocos los estudios que evalúan las características de la población sin trastorno mental diagnosticable y que determinan las características diferenciales de estos pacientes con los diagnosticados de 
ORIGINALES Y REVISIONES

trastorno mental. Los trabajos realizados hasta ahora son dos estadounidenses (23, 24), uno canadiense (29) y otro holandés (30)

El propósito de este trabajo es conocer si existen diferencias, y cómo son, entre las variables sociodemográficas, clínicas y asistenciales de pacientes diagnosticados de código $\mathrm{Z}$ y los diagnosticados de trastorno mental.

\section{Material y métodos}

Diseño: Estudio comparativo descriptivo.

Emplazamiento: Este estudio se realizó en el Centro de salud mental (CSM) del Distrito de Salamanca que pertenece al Área Sanitaria 2 de Madrid atiende a una población urbana de 140.000 habitantes.

Población: Se incluyeron a todos los pacientes mayores de 18 años derivados al CSM durante un año completo que no tenían historia clínica abierta en el CSM o si la tenían, habían transcurrido más de dos años desde su último contacto en el centro. Se citaron 1187 pacientes, de los que 1004 acudieron a la primera cita.

La derivación desde Atención Primaria (AP) se realiza cumplimentando un volante por el médico de Familia, en el que se especifica si solicita una cita preferente (en menos de dos semanas) o normal (entre uno y dos meses de espera), por defecto, se cita como normal. Excepcionalmente se reciben derivaciones desde el Hospital de referencia, otros servicios médicos especializados, Servicios Sociales del distrito o de los juzgados. De los 1004 pacientes que acudieron a la primera cita, 244 pacientes no tenían ningún trastorno mental diagnosticable. De los 760 pacientes restantes se seleccionó otra muestra de similar tamaño mediante un muestreo aleatorio simple para obtener la población de pacientes con trastorno mental diagnosticable y así poder realizar el análisis comparativo.

Mediciones e intervenciones: En la primera cita en el CSM (cita de evaluación) previo a la consulta, se invita a los pacientes a participar en el estudio a través de un consentimiento informado. Se les entrega los cuestionarios General Health Questionnaire-28 (GHQ-28) (31), el Symptom Cheklist 90 Revised (SCL90-R) (32), el cuestionario de acontecimientos vitales (CSV) (33) y una escala de expectativas tipo Likert puntuada de 0 (nada) a 10 (completamente) en la que se le pedía al paciente: "Señale con una raya cuánto cree que va a mejorar al venir a este Centro de salud mental"; para ser autocumplimentados en la sala de espera.

A continuación, se realiza una entrevista clínica de una hora por uno de los cinco psiquiatras o dos psicólogos del equipo del CSM según la práctica asistencial habitual. Se emite un juicio diagnóstico, se clasifica a los pacientes como trastornos mentales o no según la CIE-10, y se decide la actitud terapéutica de alta o 
seguimiento. Previamente, se consensuaron los criterios CIE-10, se delimitó qué se consideraba trastorno mental con todos los profesionales implicados y se realizó un estudio piloto durante 3 meses.

De todos los pacientes se recogían variables: a) sociodemográficas (sexo, edad, estado civil, convivencia, nivel educativo, situación laboral), b) asistenciales (tipo y sexo del profesional, tipo de derivación, equipo que deriva) y c) clínicas (antecedentes psiquiátricos, tratamiento inicial, indicación tras evaluación)

Estrategia de análisis:

El análisis estadístico se realizó en tres etapas. En primer lugar se llevó a cabo la descripción de las variables, presentando la frecuencia y porcentaje en el caso de las variables categóricas, la media, la desviación típica, la mediana y el rango para las variables cuantitativas para cada una de las dos muestras. En segundo lugar se realizó un análisis comparativo de las dos muestras. Para ello se emplea t de Student para muestras independientes para la comparación de variables cuantitativas y chi cuadrado para variables categóricas. Los resultados de estos dos pasos se presentan de manera conjunta. En tercer lugar se realizó un análisis estratificado para las variables que recogen las expectativas de los pacientes y la indicación de alta o seguimiento tras la evaluación con el objeto de identificar factores de confusión o modificadores del efecto.

\section{Resultados}

La prevalencia en nuestro estudio para los códigos $\mathrm{Z}$ es de $24,4 \%$ y de $76,6 \%$ para los trastornos mentales. Hay más solteros entre los trastornos mentales que entre los códigos Z (50,4\% frente a 38,1\%). Encontramos más personas analfabetas entre los trastornos mentales ( $9 \%$ frente a $1,6 \%$ para códigos Z) y menor porcentaje en el resto de las categorías de estudios de nivel medio y superior. No se han observado diferencias ni en la edad, el sexo, la convivencia ni la situación laboral entre las dos poblaciones (tabla 1).

Hay más pacientes entre los trastornos mentales diagnosticados por profesionales mujeres $(57,3 \%)$ que por hombres $(42,6 \%)$ mientras que para los códigos $\mathrm{Z}$ sucede lo contrario (43\% frente a 57/\%). Hay más derivaciones preferentes entre los trastornos mentales $(31,1 \%$ frente a $29,9 \%)$. No hay diferencias entre psiquiatras y psicólogos en la frecuencia con la que diagnostican códigos $\mathrm{Z}$ y trastornos mentales. Respecto al servicio que deriva no existen diferencias y se derivan en la misma proporción a códigos $\mathrm{Z}$ y trastornos mentales (tabla 2).

Las expectativas respecto al tratamiento en el Centro de Salud Mental fueron rellenadas por 193 pacientes con código Z (80\%) y 184 pacientes con trastorno 
ORIGINALES Y REVISIONES

Tabla 1

Comparación de las variables cualitativas sociodemográficas de los códigos Z y los trastornos mentales.

\begin{tabular}{|c|c|c|c|c|c|c|}
\hline \multirow{2}{*}{\begin{tabular}{|l} 
\\
Variable \\
\end{tabular}} & \multirow[b]{2}{*}{ Categoría } & \multicolumn{2}{|c|}{ Código Z } & \multicolumn{2}{|c|}{ Trastorno mental } & \multirow{2}{*}{$\begin{array}{c}\mathrm{X}^{2} \\
\mathbf{p}\end{array}$} \\
\hline & & $\mathbf{n}$ & $\%$ & $\mathbf{n}$ & $\%$ & \\
\hline \multirow[t]{2}{*}{ Sexo } & Masculino & 74 & 30,3 & 86 & 35.2 & \multirow{2}{*}{0,289} \\
\hline & Femenino & 170 & 69,7 & 158 & 64,7 & \\
\hline \multirow[t]{5}{*}{ Estado Civil } & Soltero & 93 & 38,1 & 123 & 50,4 & \multirow{5}{*}{$<0,001$} \\
\hline & Casado & 86 & 35,2 & 93 & 38,1 & \\
\hline & Viudo & 27 & 11,1 & 7 & 2,8 & \\
\hline & Divorciado & 17 & 7,0 & 8 & 3,2 & \\
\hline & Separado & 21 & 8,6 & 13 & 5,3 & \\
\hline \multirow{2}{*}{ Convivencia } & Solo & 50 & 20,5 & 55 & 22,5 & \multirow{2}{*}{0,660} \\
\hline & Acompañados & 194 & 79,5 & 189 & 77,5 & \\
\hline \multirow{6}{*}{ Nivel educativo } & Analfabeto & 4 & 1,6 & 22 & 9 & \multirow{6}{*}{0,002} \\
\hline & Estudios primarios & 45 & 18,4 & 41 & 16,8 & \\
\hline & Graduado escolar & 26 & 10,7 & 39 & 15,9 & \\
\hline & Bachillerato-F.P. & 74 & 30,3 & 53 & 21,7 & \\
\hline & Diplomado & 34 & 13,9 & 33 & 13,5 & \\
\hline & Licenciado & 61 & 25,0 & 53 & 21,7 & \\
\hline \multirow[t]{11}{*}{ Situación laboral } & Trabajando & 106 & 43,4 & 97 & 39,7 & \multirow{11}{*}{0,312} \\
\hline & Buscando primer & 5 & 2,0 & 3 & 1,2 & \\
\hline & empleo & & & & & \\
\hline & Parado sin subsidio & 9 & 3,7 & 7 & 2,8 & \\
\hline & Parado con subsidio & 10 & 4,1 & 14 & 5,7 & \\
\hline & Jubilado o retirado & 37 & 15,2 & 37 & 15,1 & \\
\hline & Estudiante & 24 & 9,0 & 17 & 6,9 & \\
\hline & Ama de casa & 24 & 9,8 & 24 & 9,8 & \\
\hline & Incapacidad laboral & 27 & 11,1 & 35 & 14,3 & \\
\hline & transitoria & & & & & \\
\hline & $\begin{array}{l}\text { Incapacidad laboral } \\
\text { permanente }\end{array}$ & 2 & 0,8 & 10 & 4,1 & \\
\hline
\end{tabular}

mental (75\%). Si analizamos la variable como cuantitativa continua vemos que no hay diferencia entre las dos poblaciones, la media está en torno a 7 en ambos casos y la mediana es 7 para los códigos $\mathrm{Z}$ y 8 para los trastornos mentales $(\mathrm{t}=1,04$, $\mathrm{p}=0,27)$. Si estudiamos la variable como cualitativa como hacen la mayoría de los estudios y agrupamos en: expectativas negativas (de 0 a 4, 5), expectativas positivas (de 5 a 8,5 ) y expectativas "mágicas" (de 9 a 10) no se observan diferencias entre las expectativas de ambos grupos $(\mathrm{p}=0,360)$. Las dos poblaciones tienden a señalar sobre todo expectativas positivas en su primera visita al CSM. En el aná- 
Tabla 2

Comparación de las variables cualitativas asistenciales de los códigos Z y los trastornos mentales.

\begin{tabular}{|c|c|c|c|c|c|c|}
\hline \multirow[b]{2}{*}{ Variable } & \multirow[b]{2}{*}{ Categoría } & \multicolumn{2}{|c|}{ Código Z } & \multicolumn{2}{|c|}{ Trastorno mental } & \multirow{2}{*}{$\begin{array}{c}\mathrm{X}^{2} \\
\mathbf{p} \\
\end{array}$} \\
\hline & & $\mathbf{n}$ & $\%$ & $\mathbf{n}$ & $\%$ & \\
\hline \multirow{2}{*}{$\begin{array}{l}\text { Tipo de } \\
\text { profesional }\end{array}$} & Psiquiatra & 187 & 77 & 199 & 81,5 & \multirow[t]{2}{*}{0,182} \\
\hline & Psicólogo & 57 & 23 & 45 & 18,4 & \\
\hline \multirow{2}{*}{$\begin{array}{l}\text { Sexo del } \\
\text { profesional }\end{array}$} & Hombre & 139 & 57 & 104 & 42,6 & \multirow[t]{2}{*}{0,002} \\
\hline & Mujer & 105 & 43 & 140 & 57,3 & \\
\hline \multirow[t]{2}{*}{ Derivación } & Ordinario & 193 & 79,1 & 168 & 68,8 & \multirow[t]{2}{*}{0,013} \\
\hline & Preferente & 51 & 20,9 & 76 & 31,1 & \\
\hline \multirow{10}{*}{$\begin{array}{l}\text { Equipo } \\
\text { que deriva } \\
\text { al paciente }\end{array}$} & Don Ramón de la Cruz & 54 & 22,1 & 37 & 15,1 & \multirow[t]{10}{*}{0,504} \\
\hline & Montesa & 39 & 16,0 & 49 & 20,1 & \\
\hline & Castelló & 25 & 10,0 & 27 & 11,0 & \\
\hline & Jorge Juan & 29 & 11,9 & 34 & 13,9 & \\
\hline & Baviera & 28 & 11,5 & 20 & 8,2 & \\
\hline & Lagasca & 27 & 11,1 & 32 & 13,1 & \\
\hline & Florestán Aguilar & 25 & 10,2 & 23 & 9,4 & \\
\hline & Urgencias & 4 & 1,6 & 7 & 2,8 & \\
\hline & U.Hospit. breve & 2 & 0,8 & 4 & 1,6 & \\
\hline & Otros & 9 & 3,7 & 11 & 4,5 & \\
\hline
\end{tabular}

Tabla 3

Frecuencia de las variables cualitativas clínicas de los códigos Z y los trastornos mentales.

\begin{tabular}{|l|l|c|c|c|c|c|}
\hline \multicolumn{2}{|c|}{} & \multicolumn{2}{c|}{ Código Z } & \multicolumn{2}{c|}{ Trastorno mental } & $\mathrm{X}^{2}$ \\
\hline Variables & Categorías & $\mathbf{n}$ & $\mathbf{( \% )}$ & $\mathbf{n}$ & $\mathbf{( \% )}$ & $\mathbf{p}$ \\
\hline Antecedentes & No & 183 & 75,0 & 131 & 53,6 & \\
Psiquiátricos & Ambulatorios & 58 & 23,8 & 94 & 38,5 & $<0,001$ \\
& Hospitalarios & 2 & 0,8 & 19 & 7,7 & \\
\hline Tratamiento & No & 124 & 51,6 & 98 & 40,8 & $<0,001$ \\
inicial & $\mathrm{Si}$ & 120 & 48,4 & 142 & 59,2 & \\
\hline Indicación tras & Alta & 127 & 52,0 & 51 & 20,9 & $<0,001$ \\
\hline
\end{tabular}

lisis estratificado por edades se observa que en el caso de los códigos Z, la mayor edad se relaciona más con las expectativas mágicas y con las negativas $(\mathrm{p}=0,009)$. Para los trastornos mentales la edad no influye en las expectativas de las pacientes al venir a consulta. Los códigos $Z$ con analfabetismo tienen más expectativas "mágicas" que los trastornos mentales $(\mathrm{p}=0,025)$. Hay más expectativas positivas en los pacientes con código $Z$ derivados de manera preferente $(\mathrm{p}=0,053)$, mientras que para los trastornos mentales no se da esta relación. 
Los trastornos mentales presentan más antecedentes de tratamiento en Salud Mental (46,2\% frente a $24,6 \%$ ), acuden al centro con un tratamiento psicofarmacológico pautado más frecuentemente que los códigos Z (59,2\% frente a 48,4\%) y tras la evaluación son dados de alta menos frecuentemente que los pacientes con código Z (20,9\% y 52\% respectivamente) (tabla 3$)$.

El GHQ-28 fue rellenado por 221 pacientes con código $\mathrm{Z}$ y 212 con trastorno mental. Si agrupamos en caso psiquiátrico (puntuación de 6 o más) y no caso psiquiátrico (5 o menos) no hay diferencias, ambos puntúan sobre todo como caso psiquiátrico $(\mathrm{p}=0,93)$.

El SCL-90- $\mathrm{R}$ fue rellenado por 188 pacientes con código $\mathrm{Z}$ y 189 pacientes con trastorno mental. Existen diferencias significativas entre todos los índices es-

Tabla 4

Comparación de los códigos Z y los trastornos mentales según el SCL-90-R.

\begin{tabular}{|c|c|c|c|c|}
\hline Índices & Códigos Z & Tr. Mentales & Diferencia (IC95\%) & Estadísticos \\
\hline Índice global de gravedad & 1,2 & 1,5 & $\begin{array}{c}0,3 \\
(0,1-0,4)\end{array}$ & $\begin{array}{c}\mathrm{t}=4,02 \\
\mathrm{p}<0,001\end{array}$ \\
\hline Total de síntomas positivos & 36,8 & 28,8 & $\begin{array}{c}7,9 \\
(13,2-25,9)\end{array}$ & $\begin{array}{c}\mathrm{t}=2,95 \\
\mathrm{p}=0,004\end{array}$ \\
\hline İndice distrés de síntomas positivos & 2,1 & 2,4 & $\begin{array}{c}0,3 \\
(0,1-0,4)\end{array}$ & $\begin{array}{c}t=4,54 \\
p=0,001\end{array}$ \\
\hline Somatización & 1,3 & 1,6 & $\begin{array}{c}0,2 \\
(0,07-0,4)\end{array}$ & $\begin{array}{l}\mathrm{t}=2,71 \\
\mathrm{p}=0,007\end{array}$ \\
\hline Obsesionalidad & 1,4 & 1,7 & $\begin{array}{c}0,3 \\
(0,1-0,5)\end{array}$ & $\begin{array}{c}\mathrm{t}=3,54 \\
\mathrm{p}<0,001\end{array}$ \\
\hline Hipersensibilidad & 1,1 & 1,3 & $\begin{array}{c}0,2 \\
(0,07-0,4)\end{array}$ & $\begin{array}{c}\mathrm{t}=2,72 \\
\mathrm{p}=0,007\end{array}$ \\
\hline Depresión & 1,7 & 2,0 & $\begin{array}{c}0,3 \\
(0,1-0,5)\end{array}$ & $\begin{array}{c}t=2,99 \\
p=0,003\end{array}$ \\
\hline Ansiedad & 1,3 & 1,8 & $\begin{array}{c}0,5 \\
(0,3-0,6)\end{array}$ & $\begin{array}{c}t=5,53 \\
p<0,001\end{array}$ \\
\hline Hostilidad & 1,0 & 1,3 & $\begin{array}{c}0,3 \\
(0,1-0,5)\end{array}$ & $\begin{array}{l}\mathrm{t}=3,02 \\
\mathrm{p}=0,003\end{array}$ \\
\hline Fobias & 0,6 & 1,1 & $\begin{array}{c}0,5 \\
(0,3-0,7)\end{array}$ & $\begin{array}{c}\mathrm{t}=5,63 \\
\mathrm{p}<0,001\end{array}$ \\
\hline Paranoidismo & 1,1 & 1,2 & $\begin{array}{c}0,1 \\
(0,07-0,3)\end{array}$ & $\begin{array}{c}\mathrm{t}=1,16 \\
\mathrm{p}=0,244\end{array}$ \\
\hline Psicoticismo & 0,7 & 1,0 & $\begin{array}{c}0,3 \\
(0,1-0,4)\end{array}$ & $\begin{array}{c}\mathrm{t}=4,30 \\
\mathrm{p}<0,001\end{array}$ \\
\hline Síntomas discretos & 1,5 & 1,7 & $\begin{array}{c}0,2 \\
(0,03-0,4)\end{array}$ & $\begin{aligned} \mathrm{t} & =2,29 \\
\mathrm{p} & =0,022\end{aligned}$ \\
\hline
\end{tabular}


tudiados entre las dos poblaciones a excepción del paranoidismo. Los trastornos mentales puntúan más alto en todos los índices estudiados, también puntúan mayor índice global de gravedad y de distrés de síntomas positivos y menor número total de síntomas positivos (tabla 4).

El cuestionario de acontecimientos vitales fue rellenado por 173 pacientes con código $\mathrm{Z}$ y 152 pacientes con trastorno mental. No se encontró diferencia en el número de sucesos vitales que señalan códigos $\mathrm{Z}$ y trastornos mentales. Los $\mathrm{Z}$ señalaban una media de 5,2 sucesos y los trastornos mentales del 5,8. En las dos poblaciones encontramos que los cambios en el ritmo de sueño es lo más frecuentemente señalado (38\% de códigos $\mathrm{Z}$ y $44 \%$ de trastorno mental). No existe gran variabilidad entre lo señalado más comúnmente por los códigos $\mathrm{Z}$ y los trastornos mentales, ambos subgrupos señalan acontecimientos vitales como preocupación por enfermedad de uno mismo o de otro miembro de la familia, malestar por la pérdida de empleo, o problemas con los colegas o compañeros de trabajo entre otros. En cuanto a las características de los sucesos vitales estresantes, el grado de estrés con el que es vivido el suceso vital es similar para ambas poblaciones, que señalan sobre todo mucho (32 y 30\%) o muchísimo estrés (30 y 36\% respectivamente). Tampoco hay diferencia en la calificación del estrés que fue considerada negativa en su mayor parte por las dos poblaciones (67\%). También los sucesos eran esperados e inesperados casi a partes iguales por ambas poblaciones $(52,5$ y $50,1 \%$ esperados respectivamente).

En lo que sí se ha encontrado diferencia es en el sentimiento de control del estrés, los trastornos mentales sentían que los acontecimientos vitales estaban fuera de control en más ocasiones (46\%) que los códigos Z (39\%) en el momento de la consulta (tabla 5).

Tabla 5

Características de los acontecimientos vitales señaladas por los códigos Z y los trastornos mentales.

\begin{tabular}{|c|c|c|c|c|c|c|}
\hline \multirow{2}{*}{\multicolumn{2}{|c|}{ Características del acontecimiento vital }} & \multicolumn{2}{|c|}{ Códigos Z } & \multicolumn{2}{|c|}{ Trastorno mental } & \multirow{2}{*}{$\begin{array}{l}X^{2} \\
\mathbf{p}\end{array}$} \\
\hline & & $\mathbf{n}$ & $\%$ & $\mathbf{n}$ & $\%$ & \\
\hline Grado de estrés & $\begin{array}{l}\text { Nada/Muy poco } \\
\text { Bastante } \\
\text { Mucho } \\
\text { Muchísimo }\end{array}$ & $\begin{array}{l}166 \\
266 \\
405 \\
433 \\
\end{array}$ & $\begin{array}{l}13 \\
21 \\
32 \\
34\end{array}$ & $\begin{array}{l}186 \\
281 \\
423 \\
511 \\
\end{array}$ & $\begin{array}{l}13 \\
20 \\
30 \\
36\end{array}$ & 0,580 \\
\hline $\begin{array}{l}\text { Calificación del } \\
\text { estrés }\end{array}$ & $\begin{array}{l}\text { Positivo } \\
\text { Negativo }\end{array}$ & $\begin{array}{l}407 \\
828\end{array}$ & $\begin{array}{l}33 \\
67\end{array}$ & $\begin{array}{l}459 \\
939\end{array}$ & $\begin{array}{l}33 \\
67\end{array}$ & 0,967 \\
\hline $\begin{array}{l}\text { Sorpresa del } \\
\text { estrés }\end{array}$ & $\begin{array}{l}\text { Esperado } \\
\text { Inesperado }\end{array}$ & $\begin{array}{l}638 \\
579\end{array}$ & $\begin{array}{l}52,5 \\
47,5\end{array}$ & $\begin{array}{l}696 \\
692\end{array}$ & $\begin{array}{l}50,1 \\
49,9\end{array}$ & 0,255 \\
\hline Control del estrés & $\begin{array}{l}\text { Controlado } \\
\text { Fuera de control }\end{array}$ & $\begin{array}{l}747 \\
471\end{array}$ & $\begin{array}{l}61 \\
39\end{array}$ & $\begin{array}{l}755 \\
640\end{array}$ & $\begin{array}{l}54 \\
46\end{array}$ & $<0,001$ \\
\hline
\end{tabular}


ORIGINALES Y REVISIONES

\section{Discusión}

Los pacientes diagnosticados de trastorno mental están solteros en mayor proporción, tienen menor nivel educativo que los códigos $\mathrm{Z}$ y están de baja o tienen incapacidades laborales permanentes en más ocasiones. En alguna ocasión se ha señalado que el diagnosticar como códigos $\mathrm{Z}$ a personas con características sociodemográficas ventajosas (estar casado, con alto nivel educativo, estar trabajando y vivir con otros) podría tener que ver con el fenómeno de "etiquetado social", ya que se supone que son personas con más recursos personales y más apoyo a nivel social (34). Sin embargo, tanto en nuestro estudio como en el resto de la literatura, aparecen algunas diferencias entre las dos poblaciones, aunque su potencia como predictoras del diagnóstico es muy débil. La educación y el empleo son las variables con mayor asociación al diagnóstico en casi todos los estudios $(23,24,29,30)$.

En nuestro estudio se observa que existen diferencias significativas en cuanto a cómo son derivados los códigos Z: la derivación normal/preferente sigue una razón 4/1 mientras que en el caso de los trastornos mentales es de 2/1. Esto significa que los trastornos mentales están ocupando más huecos de preferentes, lo cual puede ser un indicador de buen funcionamiento asistencial. Sin embargo, llama la atención que un $20 \%$ de los sujetos diagnosticados de código Z ocupa también huecos de preferentes a pesar de que se trata de pacientes que no tienen ninguna patología mental.

No existen diferencias en cuanto a los centros de atención primaria que derivan entre las dos poblaciones. Posiblemente, más allá de las características particulares de cada centro a la hora de derivar pueden influir las características de los pacientes y de los profesionales a la hora de canalizar el filtro de AP.

En cuanto a las expectativas hacia el tratamiento en Salud Mental, ambas poblaciones tenían sobre todo expectativas positivas en un $50 \%$ aproximadamente, después le seguían en proporción las expectativas mágicas y por último las negativas. En los pacientes diagnosticados de código $\mathrm{Z}$ existe mayor modulación de las expectativas que el paciente trae a consulta según algunas características. Los pacientes con código $\mathrm{Z}$ tienen más expectativas positivas hacia el tratamiento en el centro de salud mental en la medida que son más jóvenes, tienen mayor nivel educativo y son derivados de forma preferente. Los códigos $\mathrm{Z}$ tienen más expectativas mágicas cuanto mayor sea su edad, menor sea su nivel educativo y si son derivados con carácter normal. Este fenómeno va a condicionar probablemente la intervención que se lleve a cabo con estos pacientes, pues las expectativas que el paciente trae influirán en buena medida en el encuentro del profesional con el paciente. Parece que en los pacientes diagnosticados de trastorno mental las características sociodemográficas y asistenciales no influyen tanto en lo que esperan del tratamiento que puedan realizar en el centro de salud mental. 
Los trastornos mentales tienen contactos ambulatorios en Salud Mental $(38,5 \%)$ y hospitalarios $(7,7 \%)$ en mayor proporción que los códigos Z $(23,8 \%$ ambulatorios y $0,8 \%$ hospitalarios), al igual que se observa en los estudios realizados anteriormente. Llama la atención no obstante, que tanto los códigos $\mathrm{Z}$ como los trastornos mentales de los otros estudios tienen más antecedentes psiquiátricos ambulatorios y hospitalarios ( $30 \%$ para códigos $\mathrm{Z}$ y $80 \%$ para trastornos mentales en el de Windle (23), 50\% y 56\% respectivamente en el de Siddique CM and Aubry TD (29), $49 \%$ y $64 \%$ en el de Spinhoven P (30) y 40,3\% y 68,4\% en el de Figuereido JM (24) La diferencia entre los trastornos mentales y los códigos Z, en cuanto al tratamiento en Salud Mental, es mucho menor que en nuestro estudio, ya que nuestros pacientes con código Z tienen muchos menos antecedentes de tratamiento en Salud Mental. Esto quizá podría explicarse por la diferencia entre sistemas sanitarios, ya que nuestros pacientes tienen que pasar el filtro de atención primaria, y esto haría que se frenasen más las demandas que provienen de pacientes que serían diagnosticados de código $\mathrm{Z}$, lo que se traduciría en un menor número de contactos previos en salud mental de esta población.

El tratamiento farmacológico inicial con el que las dos poblaciones de nuestro estudio llegan a consulta es diferente cuantitativa y cualitativamente, aunque no existe mucha diferencia en los porcentajes que alcanzan. Siddique y colaboradores encontraron que muy pocos códigos Z (11\%) tenían tratamiento psicofarmacológico pautado en el momento de la evaluación, y eran benzodiacepinas sobre todo (8\%). Los trastornos mentales tenían tratamiento en mayor proporción (48\%) y eran benzodiacepinas y neurolépticos/antidepresivos en un porcentaje parecido (23 y $25 \%$ ). La misma diferencia encuentra Spinhoven en su estudio, solo el $13 \%$ de códigos Z traen tratamiento farmacológico frente al $49 \%$ de trastornos mentales. En ambos casos predomina el tratamiento con benzodiacepinas (10\% para los códigos $\mathrm{Z}$ y $25 \%$ para los trastornos mentales). Con estos resultados podemos concluir que en nuestro estudio los pacientes llegan con más medicación pautada en el momento de la consulta sobre todo en el caso de los códigos Z, pero debemos tener en cuenta varias cuestiones. Nuestros pacientes han sido valorados por un médico en todos los casos, que en un primer momento puede hacer una prescripción farmacológica, y los estudios americanos tienen un porcentaje muy elevado de pacientes que se autoderivan, con lo cual no es tan elevada la posibilidad de que tengan un tratamiento prescrito en la primera consulta. Por otro lado, nuestro estudio es el más moderno y en los últimos años estamos asistiendo a un gran incremento en la prescripción de tratamientos antidepresivos, propiciado por la enorme popularidad que estos tratamientos han alcanzado actualmente $(35,36,37)$.

En nuestro estudio, tras ser evaluados en la primera consulta los códigos $\mathrm{Z}$ son dados de alta en mayor proporción que los trastornos mentales $(52 \%$ frente al $20,9 \%)$. En el estudio llevado a cabo por Siddique en tres centros de salud mental 
ORIGINALES Y REVISIONES

comunitarios en Canadá el 37\% de los códigos Z fueron dados de alta y el 32\% de los trastornos mentales. Spinhoven por su parte encontró que sólo el $11 \%$ de los códigos $\mathrm{Z}$ fueron dados de alta tras la primera consulta y un 7\% de los trastornos mentales. Podemos ver que nuestras cifras respecto al alta son más elevadas que en los otros estudios sobre todo en lo que respecta a los códigos Z $\mathrm{Z}$. La diferencia se hace más llamativa si tenemos en cuenta que en nuestro sistema no se permite la autoderivación y los pacientes vienen "filtrados" por otro profesional sanitario. Además existe una diferencia mayor entre las altas dadas a trastornos mentales y códigos $\mathrm{Z}$ en nuestro estudio que la que obtiene el estudio canadiense y holandés. Podríamos esperar que por ser nuestro sistema sanitario de carácter público y por soportar una importante presión asistencial se tendiera más a dar el alta a pacientes en los que no queda clara la necesidad de tratamiento psiquiátrico o psicológico ambulatorio que en el sistema americano de carácter privado, pero vemos que en el estudio holandés que posee un sistema sanitario similar al nuestro, el número de altas de pacientes con código $\mathrm{Z}$ es mucho menor. Probablemente las características asistenciales y organizativas tanto de los sistemas de Salud como las condiciones locales en particular de cada centro explicarían las diferencias.

Los códigos $\mathrm{Z}$ son dados de alta con mayor frecuencia que los trastornos mentales tras su evaluación y parece que esto no está modulado por ninguna variable. Podíamos deducir entonces que es el propio diagnóstico el que influye en que el paciente realice o no seguimiento, más allá de sus características sociodemográficas, asistenciales o clínicas.

Ambas poblaciones puntúan como caso psiquiátrico de manera idéntica, en un $84 \%$, según el GHQ-28. Este test es un instrumento de cribaje de trastornos mentales concebido para utilizar en población general y esto podría explicar por qué en nuestro estudio este test no nos sirve para diferenciar las dos poblaciones. Por otra parte, que los códigos $\mathrm{Z}$ puntúen como trastorno mental puede explicar cómo, aunque los códigos $\mathrm{Z}$ no reúnan criterios de enfermedad mental, si trasmiten un malestar importante que hace que el médico de atención primaria los derive a salud mental.

Solo hemos encontrado un estudio (30) que haya analizado la diferencia existente entre las dos poblaciones en cuanto al SCL-90. Este estudio, como el nuestro, encuentra diferencias significativas en la puntuación entre trastornos mentales y códigos Z. Además, su población de trastornos mentales obtiene una puntuación más elevada en todos los índices que los de nuestro estudio. Curiosamente, los índices de los pacientes diagnosticados de código $\mathrm{Z}$ en su estudio (incluido el índice global de gravedad) son mayores que los de nuestros trastornos mentales, a excepción del índice que mide la hostilidad y el de las fobias. Parece que la población de este estudio holandés es más sensible a la percepción de sus síntomas tratándose de un test autoaplicado. 
Si comparamos nuestros resultados con los obtenidos en otras dos muestras españolas, una de población general, (38), y otra de pacientes nuevos que acuden a consulta psiquiátrica ambulatoria (39), comprobamos que todos los índices de los códigos Z se encuentran entre las dos poblaciones, lo que indica que los códigos $\mathrm{Z}$ presentan más síntomas que la población general pero menos que el total de los pacientes que acuden al centro de salud mental. Esto nos podría hacer pensar a que los códigos $\mathrm{Z}$ tienen más síntomas que la población general y por eso consultan, pero su intensidad clínica los sitúa fuera del grupo de los trastornos mentales. Los trastornos mentales de nuestro estudio tienen índices muy parecidos al de los pacientes del estudio de González Rivera, puntúan ligeramente por debajo en todos los índices a excepción del que mide la obsesionabilidad y la ansiedad. Es bastante inferior en nuestro estudio el índice global de gravedad y el total de síntomas positivos.

Existen numerosos trabajos que señalan la relación entre los acontecimientos vitales y la aparición de malestar emocional, síntomas psiquiátricos aislados e incluso trastornos mentales $(40,41,42)$. En nuestro estudio hemos encontrado que el número de sucesos vitales que señalan trastornos mentales y códigos $\mathrm{Z}$ eran similares. Algunos autores también coinciden en el hallazgo de similar número de factores estresantes en ambas poblaciones y opinan que la diferencia está en el tipo de acontecimiento vital estresante (43). Otros señalan mayor número de acontecimientos vitales estresantes durante los últimos seis meses entre los trastornos mentales en comparación con una cohorte de pacientes que no poseían ningún trastorno (44).

Por otra parte, no existían diferencias en el grado de estrés con el que estos sucesos vitales eran vividos, ni en que fueran catalogados como negativos en su mayoría, ni en el hecho de que los acontecimientos sean esperables o no. Quizá este sea el motivo por el que los códigos Z, a pesar de no reunir criterios de enfermedad mental, traspasan el filtro de atención primaria y son derivados a salud mental. Es posible que trasmitan mayor malestar en el manejo de sus circunstancias vitales que la población general, no obstante, sí se halló que estos códigos $\mathrm{Z}$ tenían mayor proporción de estrés controlado que fuera de control en el momento de la consulta que los trastornos mentales. Quizá este podría ser un factor diferenciador y clave para establecer una frontera entre estas dos poblaciones: puede que los trastornos mentales tengan menos capacidades o habilidades de afrontamiento de las circunstancias adversas y sean por tanto más vulnerables. Existen en la literatura estudios que analizan la relación entre los recursos de afrontamiento al estrés y la existencia o no de trastorno mental $(45,46,47)$ En general coinciden en señalar menos estrategias de afrontamiento o más deficitarias (entendiéndolo como el uso de más métodos de afrontamiento a corto que a largo plazo) entre los trastornos mentales. En un estudio que comparaba población sana con pacientes diagnosticados de tras- 
ORIGINALES Y REVISIONES

torno de pánico encontraban que la diferencia entre ambas poblaciones no era el número de factores estresantes vividos en años previos, sino otras dos variables: el tipo de acontecimiento vital estresante y el uso de estrategias de afrontamiento consideradas inefectivas (escapada, evitación,...) que eran más frecuentes entre los trastornos mentales. Conclusiones similares se repiten en otros estudios $(47,48,49$, $50)$.

Hemos hallado algunos parámetros diferenciadores de las dos poblaciones, no obstante, este estudio ha sido realizado en un solo centro de salud mental a lo largo de un año entero con una muestra completa y consecutiva, lo que le proporciona una validez interna elevada, pero limita su generalización a otros servicios con una población distinta y unos centros de atención primaria y profesionales de salud mental de otras características. Sería deseable que se realizaran estudios multicéntricos para comparar los resultados, conocer mejor el impacto asistencial de la atención a estas dos poblaciones y si su discriminación más nítida permite un mejor aprovechamiento de los recursos. Para ello también es importante conocer la evolución clínica de los códigos $\mathrm{Z}$ y saber de qué tipo de intervenciones se benefician para ajustar la oferta asistencial sin que vaya en detrimento de la atención de los trastornos mentales, en especial los más graves.

\section{BIBLIOGRAFÍA:}

(1) Retolaza A ¿Salud mental o psiquiatría?: entre la atención primaria y el estigma del manicomio. Norte de Salud Mental 2004; 19, 49-57.

(2) Kessler RC, Demler O, Frank RG, Ofson M, Pincus HA, Walters EE, Wang MP, Wells KB, Zaslavsky AM Prevalence and Treatment of Mental Disorders, 1990 to 2003. N Engl J Med 2005; 352, 24, 2515-23.

(3) Angst J, Gamma A, Neuenschawander M, Ajdacic-Gross V, Eich D, Rossler W, Merikangas KR Prevalence of mental disorders in the Zurich Cohort Study: a twenty year prospective study. Epidemiol Psichiatr Soc 2005; 14, 2, 68-75.

(4) Ortiz Lobo A, García Moratalla B, Lozano Serrano C, De la Mata Ruiz I, Rodríguez Salvanés F Conditions that do not reach the threshold for mental disorder in Spanish psychiatric outpatients: prevalence, treatment and management. Int J Soc Psychiatry. published on line before print April 29, 2010 doi:10.1177/0020764010368616

(5) American Psychiatric Association Manual diagnóstico y estadístico de los trastornos mentales. Barcelona: Masson 1995

(6) Organización Mundial de la Salud Clasificación de la CIE-10 de los trastornos mentales y del comportamiento: descripciones clínicas y pautas para el diagnóstico. Madrid: Meditor 1992

(7) Magruder KM, Calderone GE, Public health consequences of different thresholds for the diagnosis of mental disorders. Compr Psychiatry 2000; 41, 14-8.

(8) Lewinson PM, Shankman SA, GauJM, Klein DN The prevalence and comorbidity of subthreshold psychiatric conditions. Psychol Med 2004; 34, 613-22. 
(9) Wakefield JC, Spitzer RL Lowered estimates- but of what? Arch Gen Psychiatry 2002; 59, 416

(10) Kessler RC, Merikangas KR, Beglund P, Eaton WW, Koretz DS, Walters EE Mild disorders should not be eliminated from the DSM-V. Arch Gen Psychiatry 2003; 60, 1117-22.

(11) Márquez S, Meneu R La medicalización de la vida y sus protagonistas. Gestión Clínica y Sanitaria 2003; 5 (2), 47-53.

(12) Moynihan R, Health I, Henry D Selling sickness: the pharmaceutical industry and disease mongering. BMJ 2002; 324, 886-891

(13) Mata Ruiz I, Ortiz Lobo A Industria farmacéutica y psiquiatría. Rev Asoc Esp Neuropsiq $2003 ; 86,49-71$.

(14) Jacobs DG National Depression Screening Day: educating the public, reaching those in need of treatment, and broadening professional understanding. Harvard Review Psychiatry 1995; 3, 156-159.

(15) Sturm R Tracking changes in behavioral health services: how have carve-outs changed care? J Behav Health Serv Res 1999; 26: 360-371.

(16) Langsley DG The community mental health centre: does it treat patients? Hosp Community Psychiatry $1980 ; 31,815-819$.

(17) Winslow WW Changing trends in CMHCs: Key to survival in the eighties. Hosp Community Psychiatry 1982; 33, 273-277.

(18) Goldman HH, Reider DA, Taube CA et al Community mental health centres and the treatment of severe mental disorders. Am J Psichiatry 1980; 137, 83-86.

(19) Jerrel JM, Larsen Jk Community mental health services in transition: who is benefiting? Am J Orthopsychiatry 1986; 56, 78-88.

(20) Klerman Gl Trends in utilization of mental health services: perspectives for health services research. Medical Care 1985; 23, 584-597.

(21) Dowell DA, Ciarlo JA Overview of the community mental health centres program from an evaluation perspective. Community Ment Health J 1983; 19, 95-125.

(22) Okin RL How Community mental health centres are coping. Hosp Community Psychiatry 1984; 35, 1118-1125

(23) Windle C, Thompson JW, Goldman HH, et al Treatment of patients with no diagnosable mental disorders in CHMCs. Hosp Community Psychiatry 1988; 39, 753-758.

(24) De Figueiredo JM, Boerstler H, O'Conell L Conditions not attributable to a mental disorder: an epidemiologic study of family problems. Am J Psychiatry 1991; 148, 780-783.

(25) Mazzoli M Conditions not attributable to a mental disorder (letter). Am J Psychiatry 1992; $149,423$.

(26) Patmore C, WeaverT Missing the CMHC bus? Nurs Times 1991; 87, $32-34$

(27) Wasylenki D, Goering P, Machaughton E Planning mental health services. I. Background and Key issues.Can J Psychiatry 1992; 37, 199-206.

(28) Rachils M, Kushner C Second opinion: what's wong with Canada's health care-system and how to fix it. Toronto: Collins Publisher 1989.

(29) Siddique CM, Aubry TD, Mulhall D The burden of conditions not attributable to mental disorders. Am J Psychiatry 1996; 153, 1489-1491.

(30) Spinhoven P, Van Der Does JW Conditions not attributable to a mental disorder in Dutch psychiatric outpatients. Psychol Med 1999; 29, 213-220. 
ORIGINALES Y REVISIONES

(31) Goldberg DP, Hillier VF A scaled version of the General Health Questionnaire. Psychol Med 1979; 9, 139-145.

(32) Derogatis LR SCL-90-R, administration, scoring and procedures manual for the Revised. Version. Baltimore: Johns Hopkins University, School of Medicine 1977

(33) Sandin B, Charot P Cuestionario de sucesos vitales. CSV UNED: Madrid 1987.

(34) Schur E Labeling deviant behavior: Its sociological implications. New York. Harper \& Row 1971

(35) Moncrieff, J Are antidepressants overrated? A review of methodological problems in antidepressant trials. J Nerv Ment Dis 2001; 189, 288-295.

(36) Gury, M IMS reports 12 percent growth in 2001 audited global pharmaceuticals sales. IMS Health 2002.

http:/www.ims-global.com/insight/news_story/0103/news_story_010314.htm

(37) Ortiz Lobo A, Lozano Serrano C El incremento en la prescripción de antidepresivos. Aten Primaria 2005; 35 (3), 125-5.

(38) De las Cuevas C, González de Rivera JL, Henry Benítez M Perfil sintomático y diagnóstico en pacientes psiquiátricos ambulatorios. Psiquis 1991; 12, 19-29.

(39) González de Rivera JL, De las Cuevas C, Gracia Marco R, et al Morbilidad psiquiátrica menor en la población general de Tenerife. Psiquis 2004; 11, 11-22.

(40) Jacobs DG National Depression Screening Day: educating the public, reaching those in need of treatment, and broadening professional understanding. Harvard Review Psychiatry 1995; 3, 156-159.

(41) Zimmermann-Tansella Ch, Donini S, Lattanzi M, et al Life events, social problems and physical health status as predictors of emotional distress in men and women in a community setting. Psychol Med 1991; 21, 505-513.

(42) Barra- Almagia Enrique Influence of stress and Depressed Mood on Adolescent Health: concurrent and Prospective analysis. Universitas Psychologica 2009 V. 8 No 1 Enero-Abril.

(43) Bell J Stressful life events and coping methods in mental-illness and -wellness behaviors, Nurs Res 1977; 26, 2, 136-41

(44) Spila B, Makara M, Chuchra M, Pawlowska B Connection between stressful live events with mental disorders. Psychiatria Polska 2005; 39, 1, 115-23.

(45) Gentil Savoia M, Bernik M, Adverse life events and coping skills in panic disorder, Revista da Hospital das Clinicas 2004; 59, 6.

(46) Katerndahl DA, Talamantes M A comparison of persons with early- versus late-onset panic attacks. J Clin Psychiatry 2000; 61:422-7.

(47) San Juan P, Magallanes A Estilos explicativos y estrategias de afrontamiento. Clínica y Salud 2007; vol 18, n 1 .

(48) Cox BJ, Endl N, Swhinson RP, Norton GR Situations and specific coping strategies associated with clinical and non-clinical panic attacks. Behav Res Ther 1992; 30:67-9.

(49) Vollrath M, Angst J Coping and illness behaviour among young adults with panic. The J Nerv Men Dis 1993; 181:303-8.

(50) Lin Qiu Biaobin Yan Stressful Life Events, Coping Strategies and Mental Health Problems among Chinese Vocational College Students This paper appears in: Bioinformatics and Biomedical Engineering, 3rd International Conference on, Issue 11-13, June 2009; pag 1-4. 